\title{
TRANSGRESIONES Y ERRORES POTENCIALES DE MEDICACIÓN EN ESTUDIANTES DE ENFERMERÍA EN AMBIENTE SIMULADO
}

\section{TRANSGRESSIONS AND POTENTIAL MEDICATION ERRORS OF NURSING STUDENTS IN A SIMULATED ENVIRONMENT}

\author{
KAREN DOMÍNGUEZ CANCINO \\ Universidad Finis Terrae, Chile \\ CAROLA BRAVO TARÉ \\ Universidad Finis Terrae, Chile \\ KAREN VERGARA ARIAS \\ Laerdal Medical Brasil \\ ELIANA ESCUDERO \\ Universidad Diego Portales, Chile
}

\section{RESUMEN}

Introducción: Los errores de medicación son uno de los eventos de mayor relevancia en la actualidad, derivándose de la interacción entre el medio y los/as profesionales. La educación debe entrenar a los/as estudiantes para la seguridad. Objetivo: identificar transgresiones y errores potenciales de medicación en estudiantes de enfermería en los subprocesos de preparación y administración de medicamentos por vía parenteral en ambiente simulado. Método: estudio cuantitativo, descriptivo realizado en estudiantes de segundo, tercer y cuarto año de la carrera de enfermería de una Universidad chilena. Se evaluaron los subprocesos de preparación y administración a través de la aplicación de una pauta estandarizada de administración segura de medicamentos en ambiente de simulación. Se reconocieron ítems críticos (ítems de transgresiones o errores potenciales) utilizando un punto de corte del $70 \%$. Resultados: los/as estudiantes presentaban edades promedio entre 22 y 24 años, predominando el sexo femenino. Los ítems identificados como críticos en el subproceso de preparación fueron: verificación de la fecha de caducidad del medicamento, transgresión de principios de infecciones asociadas a la atención de salud y preparación de la dosis correcta. En el subproceso de administración se identificó: verificación de alergias, manejo de accesos vasculares, entrega de información e identificación de paciente. Conclusiones: a través de este estudio se identificaron transgresiones y errores potenciales que deben ser trabajados con los/ as estudiantes. La simulación clínica como metodología permite el entrenamiento de competencias orientadas a la provisión de cuidados seguros y de calidad en un ambiente real y protegido.

Palabras clave: educación en Enfermería. seguridad del paciente, errores de medicación, entrenamiento simulado.

Proyecto financiado por el Concurso Anual de Investigación de la Dirección de Investigación y Postgrado de la Universidad Finis Terrae (2017).

Correspondencia a: Karen.Dominguez@globalnursing.org 


\section{ABSTRACT}

Introduction: Medication errors are one of the most relevant events nowadays, which derive from the interaction between the environment and professionals. Education has to train students regarding safety issues. Objective: to identify nursing students' transgressions and potential medication errors in the preparation and medication administration subprocesses through parenteral infusion in a simulated environment. Method: quantitative and descriptive study, conducted on students in the second, third, and fourth year of their nursing studies at a Chilean University. We evaluated the preparation and administration subprocesses through the application of a safe standardized guideline about safe medication in a simulated environment. We recognized critical items (transgressions items or potential errors) using a cut-off point of $70 \%$. Results: the students, mostly female, were between 22 and 24 years old. The items identified as critical in the preparation subprocess were: verification of medicines' expiration dates, transgression of principles of infection associated with health care and preparation of proper dosage. In the administration subprocess, we identified: drug allergy testing, vascular accesses management, provision of information and patient identification. Conclusions: through this study, we identified transgressions and potential errors that should be worked with students. Clinical simulation as a methodology allows the training of competencies oriented to the provision of quality and safe care in a real and protected environment.

Key words Nursing Education; Patient Safety; Medication Error; Simulation Training.

\section{INTRODUCCIÓN}

El error de medicación es uno de los más comunes observados en la atención de salud (MacFie, Baudouin, \& Messer 2016). Este se define como cualquier evento prevenible que pueda causar o llevar a un uso inapropiado de la medicación o a daños en el paciente cuando este se encuentra en control de los profesionales de salud, paciente o consumidor (National Coordinating Council for Medication Error Reporting and Prevention [NCCMERP], s.f.). De acuerdo a la NCCMERP, existen al menos cuatro tipos de errores de medicación, uno de estos, el error potencial (Carreño-Dueñas, Sánchez, Bermúdez-Silva, \& Mesa 2014). El error potencial se define, de acuerdo a esta fuente refererida, como aquella circunstancia o incidente con capacidad de causar error.
Existe una alta heterogeneidad en las investigaciones que declaran datos de incidencia y prevalencia de errores de medicación propiamente tal, asunto que ha sido descrito hace décadas (Hughes \& Blegen 2008). Los reportes de prevalencia fluctúan entre 5 y $75 \%$, dependiendo del marco de medición referido, y la incidencia entre 5,1 a 967 por cada 1000 días-paciente, estimando a modo global una incidencia de 9/1000 días-paciente (Assiri et al. 2018, MacFie et al. 2016). La variabilidad se ha relacionado a la técnica para obtener dichos datos (subnotificación de eventos) y al desconocimiento por parte de los profesionales de que pudieran cometer un error (Blignaut, Coetzee, Klopper \& Ellis 2017, Hughes \& Blegen 2008). Considerando lo anterior, la literatura sobre errores potenciales es más escasa aún, y se estima que estos pueden llegar a un $55 \%$, 
por lo que organizaciones internacionales de enfermería han incentivado su notificación como una práctica para la seguridad (Asensi-Vicente, Jiménez-Ruiz \& Vizcaya-Moreno 2018, Claffey 2018).

En general, el proceso de administración de medicamentos está compuesto por al menos cinco subprocesos en los cuales pueden existir fallas, en prescripción, transcripción, preparación, administración y monitorización (Hughes \& Blegen 2008, Smeulers et al. 2015, Wang et al. 2015). Un tercio de todos los errores ocurren en las etapas de preparación y administración, actividades predominantemente de enfermería (Duarte, Stipp, da Silva \& de Oliveira 2015, Fathi et al. 2017, Smeulers et al. 2015); situación que puede variar de país en país, en parte por la informatización de los procesos (Duarte et al. 2015, Orbæk, Gaard, Fabricius, Lefevre \& Møller 2015; Stewart et al. 2018; Toffoletto, Canales, Moreira, Ordenes \& Vergara 2015).

Se ha intentado identificar las razones por las cuales los profesionales de enfermería cometen errores de medicación, destacando investigaciones que analizan el fenómeno desde modelos que involucran el ambiente y a los profesionales. Tanto el modelo Eindhoven como el de Bandura identifican claramente la interacción entre estos elementos, exponiendo a los profesionales y futuros profesionales a un ambiente de alta complejidad donde el error es un resultado frecuente (Parry, Barriball \& While 2015, Valdez, de Guzman \& Escolar-Chua 2013). Se reconoce la generación de los errores en base a uno o a la combinación de varios aspectos como las fallas técnicas, de la organización y humanas, tomando en cuenta el ambiente, las características de la persona y el comportamiento final, lo que da lugar al error o violación de reglas, esta última conocida como transgresión (Blignaut et al. 2017, Parry et al. 2015, Valdez et al. 2013). En este sentido, Parry et al. (2015) señalan que en la actualidad existe una focalización en aspectos relacionados a variables ambientales, muy característico del gerenciamiento de las instituciones de salud -dejando de lado aquellas relacionadas a las personas-, lo que resulta complejo en el entendido que los profesionales de salud son seres autónomos que deben dar cuenta de sus acciones. Desde esta perspectiva, son los profesionales, y especialmente los enfermeros, los que deben ser parte de la solución.

Durante su formación, estos profesionales abordan temas como administración de medicamentos, farmacología y verificación de procedimientos correctos como estrategia de prevención del error (paciente, medicamento, dosis, ruta, tiempo, preparación, conocimiento, pregunta, razón, consejo, educación, valoración de datos, documentación, frecuencia, historia, interacción, evaluación, información, respuesta y resultado correctos, y derecho [right] a rechazar). No obstante, el ambiente real en el que los estudiantes realizan la administración de medicamentos es impredecible y no controlado (Claffey 2018, Jones \& Treiber 2018, Simonsen, Daehlin, Johansson \& Farup 2014, Valdez et al. 2013). Por otra parte, se observa que existe un espacio entre la teoría y la práctica, ya que los estudiantes tienen pocas oportunidades para preparar y administrar medicamentos durante sus pasantías (Simonsen et al. 2014, Valdez et al. 2013).

En este contexto, estudios señalan que la simulación puede ser una estrategia efectiva para prevenir los errores de medicación, ya que permite entrenar técnicas y competencias que integran los factores hu- 
manos (Sarfati et al. 2018). Es importante que los currículos preparen al estudiante en la comprensión y se familiaricen con temas de seguridad del paciente (Latimer, Hewitt, Stanbrough \& McAndrew 2017, WHO 2009).

La escuela de enfermería de la universidad donde se realizó el estudio ha incluido la medicación segura como un asunto fundamental dentro de la formación, utilizando metodologías que aumentan la seguridad del paciente en un currículo basado en competencias con simulación clínica integrada, y estableciendo cuatro mediciones High-Stake durante el desarrollo de la carrera (Escudero, Ben-Azul \& Dominguez Cancino 2018, INACSL Standards of Best Practice: SimulationSM Simulation Design 2016). Este estudio tiene por objetivo identificar transgresiones y errores potenciales de medicación cometidos por estudiantes de enfermería en los subprocesos de preparación y administración por vía parenteral en ambiente de simulación.

\section{MATERIAL Y MÉTODOS}

Estudio cuantitativo, descriptivo, enmarcado en el proyecto "Evaluación de impacto del protocolo de administración segura de medicamentos en estudiantes de Enfermería: experiencia a un año de su implementación".

La población corresponde a todos los estudiantes de la carrera de enfermería $(\mathrm{N}=278)$. Criterios de inclusión y exclusión: se incluyeron a todos los estudiantes que cursaran Farmacología Clínica entre los años 2015 y 2017, asignatura en la que se inicia la implementación del protocolo de administración segura de medicamentos. Se excluyeron a los estudiantes que por voluntad no quisieran participar.
Las variables principales de estudio se desprenden de una pauta de observación estandarizada del proceso de administración de medicamentos por vía parenteral creada por un equipo investigador de la misma universidad (Dominguez-Cancino, Arias, Caballero \& Escudero 2019). El instrumento considera datos del estudiante (nombre, asignatura, evaluador) y una pauta que incluye todos los subprocesos considerados en la administración de medicamentos: revisión de la prescripción del medicamento, valoración, transcripción, preparación, administración, notificación de errores, seguimiento y monitorización del efecto terapéutico, colaterales y adversos, e información al paciente. Los ocho subprocesos anteriores son evaluados a través de 58 ítems con posibles respuestas "si", "no" y "no aplica", ajustados al ambiente de simulación y práctica clínica, contando con validez de aspecto y contenido. En particular, los subprocesos evaluables en ambiente de simulación de la escuela son la preparación y administración de medicamentos, considerando un total de 32 ítems y dos pausas de seguridad. Los ítems fueron separados considerando dos categorías: transgresiones y errores potenciales, como se muestra en la Tabla 1.

Se incluyeron variables demográficas y académicas obtenidas a través de las bases de datos del estudiantado posterior a la obtención del consentimiento informado. Se consideraron las variables: edad (años), sexo (masculino/femenino), nivel cursado (segundo, tercer y cuarto nivel), y la reprobación de asignaturas durante la carrera, de tipo disciplinar y farmacología puntualmente (reprueba/no reprueba).

Para la recolección de datos se incluyó el uso de las pautas en evaluaciones académicas. Se solicitó a las encargadas de asig- 
Karen Dominguez-Cancino, Carola Bravo-Taré, Karen Vergara-Arias, Eliana Escudero

TABLA 1

Transgresiones y errores potenciales de medicación

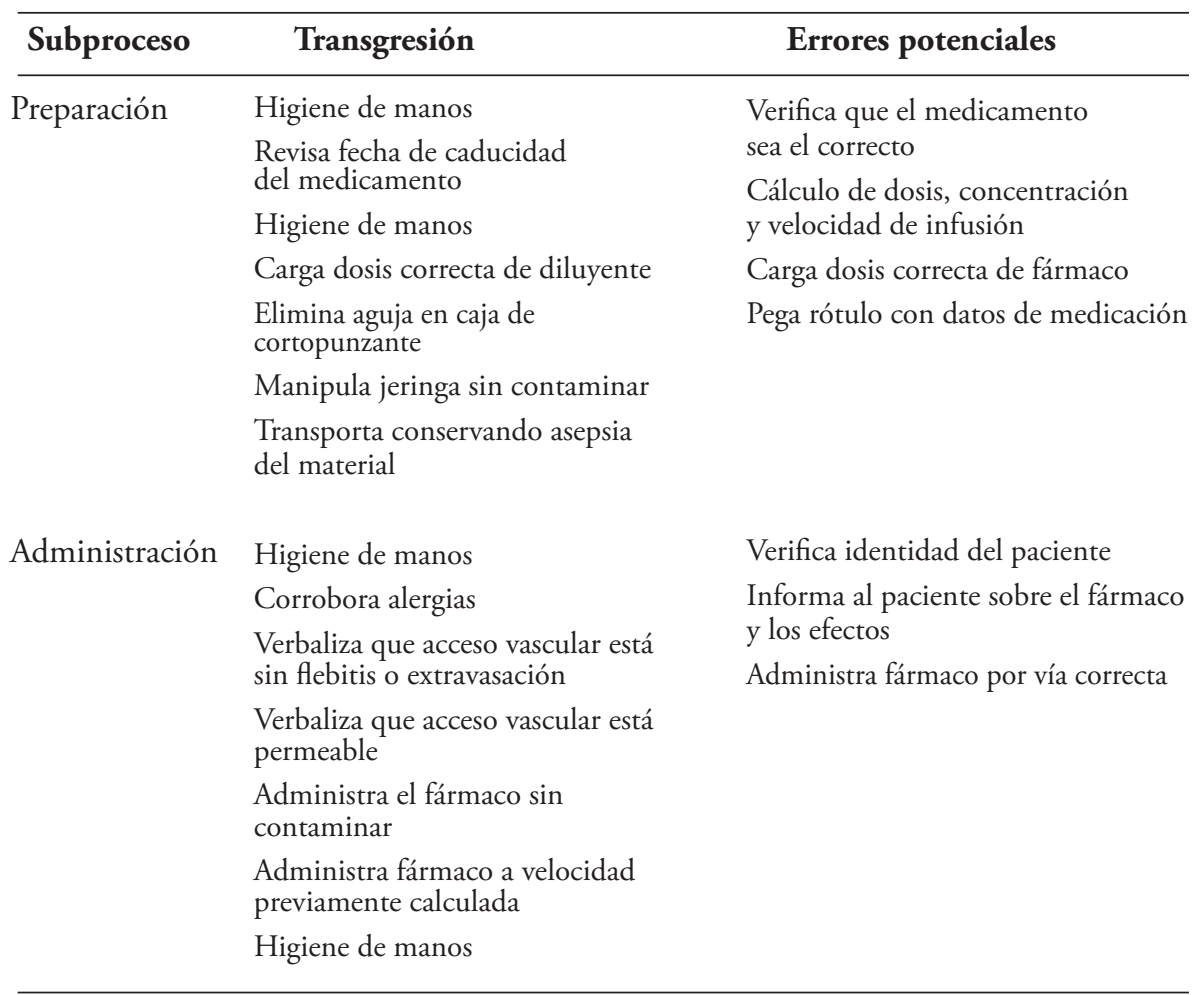

natura de enfermería médico-quirúrgica del adulto (segundo ańo de la carrera), enfermería médico-quirúrgica del niño (tercer año) y urgencias (cuarto año) utilizar los subprocesos de manera íntegra sin generar modificaciones en los ítems.

Las asignaturas anteriores cuentan con evaluaciones High-Stake posicionadas al final del semestre académico, estas evalúan de manera integrada las competencias adquiridas siendo reprobatorias (Escudero et al., 2018; "INACSL Standards of Best Practice: SimulationSM Simulation Design”, 2016).

Las tres asignaturas se encontraban en curso durante el desarrollo del proyecto del cual se desprende esta investigación y se dan en el primer semestre de cada año.

Se capacitó en el uso del instrumento a los docentes. Posterior a la aplicación de las pautas las encargadas de cada asignatura revisaban la completitud de datos y devolvían a la persona evaluadora en caso de existir algún ítem sin completar en un plazo menor a dos días. Como las pautas se encontraban enmarcadas en el desarrollo de las asignaturas, se esperó hasta el cierre de las mismas para poder acceder a los datos de los estudiantes que habían consentido su participación vía documento consentimiento informado. Las pautas fueron traspasadas a una base de datos de forma anonimizada por ayudantes de investigación. 
Se realizó estadística descriptiva a través de promedios y cuartiles (p25-p75) para variables cuantitativas, y porcentajes para variables cualitativas. Se realizó estadística bivariada de tipo no paramétrica considerando la variable nivel para comprobar que los grupos eran comparables. Se determinó el cumplimiento de cada ítem por subproceso a través del cálculo de porcentajes por cada nivel y se identificó los ítems considerados de "riesgo", es decir, aquellos que dados bajo cumplimiento pondrían en una posición de transgresión o error potencial de medicación al estudiante en la práctica clínica. Se consideró de riesgo un porcentaje menor a $70 \%$. Los análisis se realizaron a través del software estadístico STATA versión 12.0.

Para el desarrollo de la investigación se consideraron los cuatro principios bioéti$\cos$ (No maleficencia, beneficencia, justicia y autonomía), además de las normas y leyes vigentes aplicables en lo que se refiere a investigación en seres humanos. Para obtener el consentimiento informado del estudiantado se solicitó un espacio durante las asignaturas para explicar las características del estudio. El estudio obtuvo aprobación del comité de ética de la Universidad Finis Terrae (Resolución N 15/2017).

\section{RESULTADOS}

De un total de 198 estudiantes inscritos en las asignaturas de Enfermería médico-quirúrgica del adulto y Enfermería médico-quirúrgica del niño y urgencias, fueron excluidos 8 por no cumplir el criterio de iniciar el trabajo con el protocolo en el año 2015; 3, por reprobación de asignatura en el semestre anterior en contexto de ingreso a internado; y 3 , por ser ayudantes alumnos de la investigación. Y 13 rechazaron participar, constituyendo finalmente una muestra de 171 estudiantes. De estos, 65 pertenecían a segundo año; 79, a tercero; y 27 a cuarto. Cada nivel tuvo al menos una evaluación previa con la pauta estandarizada (el segundo tuvo dos mediciones previas; el tercero, cuatro; y el cuarto año, una).

La edad promedio de cada grupo de estudiantes fue de 22, 23 y 24 años; con predominancia del sexo femenino. En relación a las características académicas, la mayoría se encontraba al día en su cumplimiento curricular, solo se observó un aumento de estudiantes que no pertenecían originalmente a dicha cohorte en tercer ańo. Los porcentajes de reprobación general (alguna asignatura reprobada) se encontraba sobre el $70 \%$, mientras que los porcentajes de reprobación de asignaturas de tipo disciplinar (propias de la carrera de enfermería) y de farmacología, se encontraban bajo el $31 \%$ (véase la Tabla 2). Existieron diferencias estadísticamente significativas en las variables edad y año de ingreso.

En las tablas 3 y 4 se presenta el nivel de cumplimiento del protocolo de administración segura de medicamentos en los subprocesos de preparación y administración por vía parenteral, respectivamente; se destacan los ítems que estuvieron bajo $70 \%$ de cumplimiento. De esta forma, se observaron las transgresiones "revisar fecha de caducidad del medicamento", "manipular la jeringa sin contaminar" y el error potencial "cargar la dosis correcta del fármaco" en el subproceso de preparación. Se destaca que el $100 \%$ de los estudiantes de segundo año hubiera contaminado el medicamento por una mala manipulación de insumos, y cerca de un $90 \%$ hubiera administrado un medicamento sin saber que este había caducado. Por otra parte, aproximadamente un $50 \%$ de los estudiantes de cuarto año 
hubiera cometido un error de dosis si este hubiera llegado a un paciente real.

Para el caso de la administración de medicamentos, se identificó como transgresión "corrobora alergias", "verbaliza que el acceso vascular está sin flebitis o extravasación”, y como error potencial "informar al paciente sobre el fármaco y los efectos" y "verificar identidad del paciente". De acuerdo a esto, aproximadamente un $60 \%$ de los estu- diantes de segundo año hubiera cometido un error de paciente, y al menos un $55 \%$ de los estudiantes de segundo y cuarto año no hubiera informado al paciente sobre el fármaco y sus efectos. En el caso de las transgresiones, $40 \%$ o menos hubiera corroborado alergias y $35 \%$ o menos hubiera chequeado que el acceso vascular se encontraba en condiciones para administrar el medicamento por vía parenteral.

\section{TABLA 2}

Características sociodemográficas y académicas de la población $(\mathrm{N}=171)$

\begin{tabular}{|c|c|c|c|c|}
\hline Características & $\begin{array}{l}2 .^{\circ} \text { nivel } \\
(\mathrm{n}=65)\end{array}$ & $\begin{array}{l}3 .^{\text {er }} \text { nivel } \\
(n=79)\end{array}$ & $\begin{array}{l}4 .^{\circ} \text { nivel } \\
(\mathrm{n}=27)\end{array}$ & P value. \\
\hline Edad, X(p25-p75) & $21,95(21-23)$ & $23,50(22-24)$ & $24,37(22-25)$ & $<0,001^{*}$ \\
\hline \multicolumn{5}{|l|}{ Sexo, N (\%) } \\
\hline Masculino & $12(18,46)$ & $12(15,19)$ & $3(11,11)$ & \\
\hline Femenino & $53(81,54)$ & $67(84,81)$ & $24(88,89)$ & $0,689^{* *}$ \\
\hline \multicolumn{5}{|c|}{ Año de ingreso, $\mathrm{N}(\%)$} \\
\hline 2012 & $0(0,00)$ & $2(2,53)$ & $4(14,81)$ & \\
\hline 2013 & $3(4,62)$ & $12(15,19)$ & $6(22,22)$ & \\
\hline 2014 & $12(18,46)$ & $34(43,04)$ & $17(62,96)$ & \\
\hline 2015 & $13(20,00)$ & $31(39,24)$ & $0(0,00)$ & \\
\hline 2016 & $37(56,92)$ & $0(0,00)$ & $0(0,00)$ & $<0,001$ \\
\hline \multicolumn{5}{|l|}{ Reprobación, N(\%) } \\
\hline No & $8(12,31)$ & $15(18,99)$ & $8(29,63)$ & \\
\hline Sí & $57(87,69)$ & $64(81,01)$ & $19(70,37)$ & $0,150^{* *}$ \\
\hline \multicolumn{5}{|c|}{ Reprobación disciplinar, N(\%) } \\
\hline No & $45(69,23)$ & $64(81,01)$ & $24(88,89)$ & \\
\hline Sí & $20(30,77)$ & $15(18,99)$ & $3(11,11)$ & $0,080^{* *}$ \\
\hline \multicolumn{5}{|c|}{ Reprobación farmacología, N(\%) } \\
\hline No & $49(75,38)$ & $62(78,48)$ & $20(74,07)$ & \\
\hline Sí & $16(24,62)$ & $17(21,52)$ & $7(25,93)$ & $0,833^{* *}$ \\
\hline
\end{tabular}




\section{TABLA 3}

Nivel de cumplimiento del protocolo de administración segura de medicamentos en el subproceso de preparación

\begin{tabular}{|c|c|c|c|c|}
\hline Ítems & $\begin{array}{l}\text { Cumplimiento } \\
2 .{ }^{\circ} \text { nivel }(\%)\end{array}$ & $\begin{array}{l}\text { Cumplimiento } \\
3 \text { er }^{\text {r }} \text { nivel }(\%)\end{array}$ & $\begin{array}{l}\text { Cumplimiento } \\
4 .^{\circ} \text { nivel }(\%)\end{array}$ & Promedio \\
\hline Higiene de manos & 81,16 & 97,47 & 85,19 & 87,94 \\
\hline $\begin{array}{l}\text { Verifica que el medicamento sea } \\
\text { el correcto }\end{array}$ & 85,51 & 89,87 & 81,48 & 85,62 \\
\hline $\begin{array}{l}\text { Revisa fecha de caducidad del } \\
\text { medicamento }\end{array}$ & 8,70 & 70,89 & 37,04 & 38,87 \\
\hline $\begin{array}{l}\text { Cálculo de dosis, concentración y } \\
\text { velocidad de infusión }\end{array}$ & 82,61 & 87,34 & 88,00 & 85,98 \\
\hline Higiene de manos & 75,36 & 93,67 & 74,07 & 81,04 \\
\hline Carga dosis correcta de fármaco & 79,71 & 75,95 & 51,85 & 69,17 \\
\hline Carga dosis correcta de diluyente & 81,16 & 81,01 & 72,00 & 78,06 \\
\hline Elimina aguja en caja de cortopunzante & 88,41 & 97,47 & 92,59 & 92,82 \\
\hline Manipula jeringa sin contaminar & 0,00 & 94,94 & 80,77 & 58,57 \\
\hline Pega rótulo con datos de medicación & 78,26 & 96,20 & 80,77 & 85,08 \\
\hline Transporta conservando asepsia del material & 76,81 & 98,73 & 84,62 & 86,72 \\
\hline
\end{tabular}

TABLA 4

Nivel de cumplimiento del protocolo de administración segura de medicamentos en el subproceso de administración

\begin{tabular}{|c|c|c|c|c|}
\hline Ítems & $\begin{array}{l}\text { Cumplimiento } \\
2 .^{\circ} \text { nivel (\%) }\end{array}$ & $\begin{array}{l}\text { Cumplimiento } \\
3 .^{\text {er }} \text { nivel }(\%)\end{array}$ & $\begin{array}{l}\text { Cumplimiento } \\
4 .^{\circ} \text { nivel (\%) }\end{array}$ & Promedio \\
\hline Higiene de manos & 75,36 & 97,47 & 88,89 & 87,24 \\
\hline Verifica identidad del paciente & 42,03 & 74,68 & 88,46 & 68,39 \\
\hline $\begin{array}{l}\text { Informa al paciente sobre el fármaco } \\
\text { y los efectos }\end{array}$ & 56,52 & 74,68 & 45,83 & 59,01 \\
\hline Corrobora alergias & 8,70 & 59,49 & 19,23 & 29,14 \\
\hline $\begin{array}{l}\text { Verbaliza que acceso vascular está } \\
\text { sin flebitis o extravasación }\end{array}$ & 24,64 & 64,56 & 62,96 & 50,72 \\
\hline $\begin{array}{l}\text { Verbaliza que acceso vascular está } \\
\text { permeable }\end{array}$ & 73,91 & 93,67 & 92,59 & 86,73 \\
\hline Administra el fármaco por vía correcta & 75,36 & 93,67 & 90,91 & 86,65 \\
\hline Administra el fármaco sin contaminar & 65,22 & 79,75 & 92,59 & 79,19 \\
\hline $\begin{array}{l}\text { Administra el fármaco a velocidad } \\
\text { previamente calculada }\end{array}$ & - & 86,08 & 95,65 & 90,86 \\
\hline Higiene de manos & 0,00 & 82,28 & 88,46 & 85,37 \\
\hline
\end{tabular}


Se observó que existían diferencias entre los niveles. Los estudiantes de tercer nivel tuvieron mejores porcentajes de cumplimiento en prácticamente todos los ítems.

\section{DISCUSIÓN}

La investigación tuvo por objetivo identificar transgresiones y errores potenciales de medicación cometidos por estudiantes de enfermería en los subprocesos de preparación y administración por vía parenteral utilizando la simulación clínica. Los ítems identificados como críticos en el subproceso de preparación fueron la verificación de la fecha de caducidad del medicamento, la aplicación de principios de asepsia y antisepsia y la preparación de la dosis correcta. En el subproceso de administración se identificó: verificación de alergias, entrega de información al paciente e identificación del paciente.

Estos hallazgos no concuerdan completamente con lo observado a nivel internacional, donde se identifican como errores más prevalentes la omisión, tiempo, dosis y paciente incorrecto (Fathi et al. 2017, Wang et al. 2015). Se observa que aquellos ítems que concuerdan con lo estipulado en la literatura como los errores de dosis y paciente, se encuentran muy cercanos al punto de corte del $70 \%$. Se destaca que para identificar correctamente al paciente se solicita a los estudiantes triangular información con la ficha clínica, el brazalete y preguntándole el nombre al paciente; si no se cumple con alguno de estos puntos, se considera como ítem no cumplido.

De esta manera, se observa en general un buen desempeño del estudiantado en subprocesos claves de la administración de medicamentos. Existen limitados estudios que identifican como principales errores de medicación problemas relacionados al paciente como la presencia de una alergia conocida y la entrega de información a este (Dedefo, Mitike \& Angamo 2016; Gnädinger et al. 2017; Leahy, Lavoie, Zurakowski, Baier \& Brustowicz 2018). Respecto a las alergias, algunos investigadores señalan la importancia que tiene este tema, por lo que sugieren su inclusión dentro de los principales correctos en el proceso de medicación (Mortell 2019).

Por otra parte, investigaciones recientes han incluido la violación de protocolos en el proceso de administración de medicamentos. En este sentido, los estudios concuerdan en que la transgresión de medidas de asepsia y antisepsia son comunes tanto en profesionales como en estudiantes (Asensi-Vicente et al. 2018; Blignaut et al. 2017; Chaves, Lima, Fernandes, Matias \& Araújo 2018).

La división entre transgresiones y errores potenciales de medicación se incluyó para clarificar los resultados, sin embargo, destacamos que los ítems considerados son evaluados de igual forma, es decir, se le da el mismo valor a transgresiones y errores de manera de formar un criterio sólido respecto a la importancia que tienen todos estos puntos en la seguridad del paciente.

El buen desempeño del estudiantado puede ser explicado en base a dos aspectos claves. En primer lugar, el uso de protocolos y prácticas estandarizadas ha sido identificado como una de las estrategias más efectivas para la prevención del error, posicionándose en tercer lugar luego de las funciones forzadas y la automatización del proceso de medicación (Paparella 2008). Esto permite que los futuros profesionales puedan incluir dentro de sus acciones habituales aspectos de seguridad, incor- 
porándolos en su modelo mental. Como segundo aspecto clave, investigaciones actuales consideran dentro de sus estrategias el entrenamiento y la optimización de políticas relacionadas a la administración de medicamentos, siendo altamente efectivas en la toma de conciencia del problema por parte de los profesionales de enfermería $(\mathrm{Xu}, \mathrm{Li}, \mathrm{Ye} \& \mathrm{Lu} 2014)$. En este sentido, la simulación se ha reconocido como una estrategia educativa que permite el entrenamiento y el logro de competencias como seguridad del paciente y comunicación, valoración y diagnóstico enfermero, y aplicación de intervenciones y pensamiento crítico, tanto en estudiantes como en profesionales, permitiendo a estos practicar las intervenciones enfermeras y analizar sus resultados (Raurell-Torredà et al. 2015). Además, permite entrenar la comunicación efectiva y la toma de decisiones, aspectos claves a la hora de prevenir errores de medicación (Sarfati et al. 2018). Es por esto que la simulación clínica se considera un buen enfoque para educar en cuanto a la administración segura de medicamentos, previniendo los errores en pacientes reales (Sanko \& Mckay 2017).

Por otra parte, se indica que a medida que los estudiantes avanzan en su carrera respetan menos los protocolos, lo que explicaría lo observado en cuarto año; además, se indica que la falta de experiencia se relaciona fuertemente a los errores de medicación, lo que daría cuenta de lo ocurrido en segundo año, en especial en las transgresiones asociadas a medidas de asepsia y antisepsia (Asensi-Vicente et al. 2018).

Como debilidades del estudio se identifican el diferente entrenamiento previo de los distintos niveles, distintos evaluadores en cada nivel, y el efecto sinérgico entre la aplicación de la pauta y el entrena- miento en simulación. Respecto al primer punto, se identifica que los estudiantes de tercer año tuvieron más exposición a la pauta antes de la evaluación final, siendo un elemento importante que tributa a las diferencias observadas entre los niveles. Por otra parte, a pesar que todos los evaluadores fueron entrenados en la pauta, es conocido que el facilitador es una pieza clave en el proceso de evaluación con simulación. Según la International Nursing Association for Clinical Simulation and Learning (INACSL); el facilitador debe cumplir con ciertos criterios como conocimiento, experiencia, habilidades, entre otros (INACSL Standards of Best Practice: Simulation SM Simulation Design 2016). Finalmente, se considera una debilidad, desde la perspectiva de investigación, el haber generado una doble intervención en el proceso de formación-evaluación, ya que no se puede efectivamente aislar el efecto de ambas estrategias, dejando la duda de cuál fue la intervención con mayor peso en la generación de estrategias de prevención de error.

Se destaca que esta experiencia ocurre en un ambiente simulado, por lo que existen algunos errores que no pueden observarse en los escenarios utilizados en la escuela, como los errores de tiempo, omisión y medicamento. Esto se debe a que en el escenario se entrega una indicación médica determinada, señalando claramente el medicamento que debe administrar en ese momento puntual (la duración del escenario no se extiende por más de 15 minutos) y se deja a disposición solo el medicamento que debe administrar.

No obstante lo señalado, consideramos que este estudio tiene amplias fortalezas. En primer lugar, esta es la primera iniciativa descrita con las características antes señaladas: educación para la prevención del 
error de medicación, aplicando simulación clínica evaluada con una pauta estandarizada de administración de medicamentos que incluye aspectos fundamentales de seguridad a entrenar con los estudiantes. La identificación de los aspectos con mayor probabilidad de error en un ambiente simulado nos permite identificar áreas débiles para seguir trabajando.

De acuerdo a Valdez et al., los estudiantes que adquieren prácticas que violan las reglas van a tender a adherir poco a estrategias tan básicas como el cumplimiento de los correctos, en contraparte, aquellos que cumplen con los correctos pueden ser profesionales activos en la prevención del error, por lo que es importante reforzar constantemente el cumplimiento de normas básicas de prevención de error y la aplicación de otras estrategias que mejoran los procesos de seguridad en la atención del paciente, como la notificación de eventos y la aplicación del doble chequeo (Douglass et al. 2018; Sears, O’Brien-Pallas, Stevens \& Murphy 2013).

Como desafío se visualiza el traslado de esta experiencia a espacios reales para entrenar acciones preventivas en ambientes de alta complejidad.

\section{CONCLUSIONES}

Por medio de este estudio se identificó transgresiones en los subprocesos de preparación y administración relacionados a mantención de asepsia y antisepsia, consideración de alergias y manejo adecuado de accesos vasculares. Por otra parte, respecto a los errores potenciales se identificó administrar un medicamento a un paciente incorrecto, una dosis incorrecta y no informar correctamente al paciente respecto a la medicación entregada.
El logro de competencias en esta área implica planificar y establecer niveles de dominio tanto de conocimientos como de desempeños que requieren los profesionales de enfermería y, con ello, hacer un plan de entrenamiento estandarizado, tal como se ha hecho frente a otras situaciones de impacto universal.

\section{REFERENCIAS BIBLIOGRÁFICAS}

Asensi-Vicente J, Jiménez-Ruiz I \& Vizcaya-Moreno MF (2018, septiembre 1). Medication Errors Involving Nursing Students: A Systematic Review. Nurse Educator. Lippincott Williams and Wilkins. Disponible en: https://doi.org/10.1097/ NNE.0000000000000481.

Assiri GA, Shebl NA, Mahmoud MA, Aloudah N, Grant E, Aljadhey H \& Sheikh A (2018). What is the epidemiology of medication errors, error-related adverse events and risk factors for errors in adults managed in community care contexts? A systematic review of the international literature. BMJ Open, 8 (5), e019101. https://doi.org/10.1136/ bmjopen-2017-019101.

Blignaut AJ, Coetzee, SK, Klopper HC \& Ellis SM (2017). Medication administration errors and related deviations from safe practice: an observational study. Journal of Clinical Nursing, 26 (21-22), 36103623. https://doi.org/10.1111/ jocn. 13732 .

Carreño-Dueñas JA, Sánchez JO, Bermúdez-Silva CD \& Mesa M (2014). Errores en la formulación de quimioterapia. Revista Colombiana de Cancerología, 18 (4), 179-185. Disponible en: https://doi.org/10.1016/j. rccan.2014.08.003. 
Chaves CMP, Lima FET, Fernandes AFC, Matias ÉO \& Araújo PR (2018). Assessment of the preparation and administration of oral medications to institutionalized children. Revista brasileira de enfermagem, 71, 13881394. https://doi.org/10.1590/00347167-2017-0197.

Claffey C (2018). Near-miss medication errors provide a wake-up call. Nursing, 48 (1), 53-55. https://doi.org/10.1097/01.NURSE.0000527615.45031.9e.

Dedefo MG, Mitike AH \& Angamo M T (2016). Incidence and determinants of medication errors and adverse drug events among hospitalized children in West Ethiopia. BMC pediatrics, 16, 81. https://doi.org/10.1186/s12887016-0619-5.

Dominguez-Cancino KA, Arias M, Caballero E \& Escudero E (2019). Elaboración de una pauta de evaluación de administración segura de medicamentos en estudiantes de enfermería. Rev. Latino-Am. Enfermagem. https://doi.org/10.1590/15188345.2989.3246).

Douglass AM, Elder J, Watson R, Kallay T, Kirsh D, Robb WG, Coil C J (2018). A Randomized Controlled Trial on the Effect of a Double Check on the Detection of Medication Errors. Annals of Emergency Medicine, 71 (1), 74-82.e1. https://doi.org/10.1016/j. annemergmed.2017.03.022.

Duarte SdaCM, Stipp MAC, da Silva MM \& de Oliveira FT (2015). Adverse events and safety in nursing care. Revista Brasileira De Enfermagem, 68 (1), 136146, 144-154. https://doi.org/10.159/0034-7167.2015680120p

Escudero E, Ben-Azul MA \& Dominguez Cancino K (2018). Clinical simulation and patient safety: integra- tion into the nursing curriculum. Scientia Medica, 28(1), 28853. https://doi.org/10.15448/19806108.2018.1.28853.

Fathi A, Hajizadeh M, Moradi K, Zandian H, Dezhkameh M, Kazemzadeh, S \& Rezaei S (2017). Medication errors among nurses in teaching hospitals in the west of Iran: what we need to know about prevalence, types, and barriers to reporting. Epidemiology and health, 39, e2017022. https:// doi.org/10.4178/epih.e2017022.

Gnädinger M, Conen D, Herzig L, Puhan MA, Staehelin A, Zoller M \& Ceschi A (2017). Medication incidents in primary care medicine: a prospective study in the Swiss Sentinel Surveillance Network (Sentinella). BMJ open, 7(7), e013658. https://doi. org/10.1136/bmjopen-2016-013658.

Hughes RG \& Blegen MA (2008). Medication Administration Safety. Patient Safety and Quality: An Evidence-Based Handbook for Nurses. Agency for Healthcare Research and Quality (US). Recuperado de http://www.ncbi.nlm.nih.gov/pubmed/21328757.

INACSL Standards of Best Practice: SimulationSM Simulation Design. (2016). Clinical Simulation in Nursing, 12, S5-S12. https://doi.org/10.1016/j. ecns.2016.09.005.

Jones JH \& Treiber LA (2018). Nurses' rights of medication administration: Including authority with accountability and responsibility. Nursing Forum, 53(3), 299-303. https://doi. org/10.1111/nuf.12252.

Latimer S, Hewitt J, Stanbrough R \& McAndrew R (2017). Reducing medication errors: Teaching strategies that increase nursing students' awareness of medication errors and their prevention. Nurse Education Today, 
52, 7-9. https://doi.org/10.1016/j. nedt.2017.02.004.

Leahy IC, Lavoie M, Zurakowski D, Baier AW \& Brustowicz RM (2018). Medication errors in a pediatric anesthesia setting: Incidence, etiologies, and error reduction strategies. Journal of Clinical Anesthesia, 49, 107-111. https://doi.org/10.1016/j.jclinane.2018.05.011.

MacFie CC, Baudouin SV \& Messer PB (2016). An integrative review of drug errors in critical care. Journal of the Intensive Care Society, 17(1), 63-72. https://doi. org/10.1177/1751143715605119.

Mortell M (2019). Should known allergy status be included as a medication administration 'right'? British Journal of Nursing, 28(20), 1292 1298. https://doi.org/10.12968/ bjon.2019.28.20.1292.

National Coordinating Council for Medication Error Reporting and Prevention. (s. f.). About Medication Errors | NCC MERP. Consulta: 7 de agosto de 2018. Disponible en: http:// www.nccmerp.org/about-medication-errors

Orbæk J, Gaard M, Fabricius P, Lefevre RS \& Møller T (2015). Patient safety and technology-driven medication A qualitative study on how graduate nursing students navigate through complex medication administration. Nurse Education in Practice, 15(3), 203-211. https://doi.org/10.1016/j. nepr.2014.11.015.

Paparella S (2008). Choosing the Right Strategy for Medication Error Reduction: Part I. Journal of Emergency Nursing, 34(2), 145-146. https://doi.org/10.1016/j.jen.2007.11.011

Parry AM, Barriball KL \& While AE (2015). Factors contributing to Registered
Nurse medication administration error: A narrative review. International Journal of Nursing Studies, 52(1), 403-420. https://doi.org/10.1016/j. ijnurstu.2014.07.003.

Raurell-Torredà M, Olivet-Pujol J, Romero-Collado À, Malagon-Aguilera MC, Patiño-Masó J \& Baltasar-Bagué A (2015). Case-Based Learning and Simulation: Useful Tools to Enhance Nurses' Education? Nonrandomized Controlled Trial. Journal of Nursing Scholarship, 47(1), 34-42. https://doi.org/10.1111/jnu.12113.

Sanko JS \& Mckay M (2017). Impact of Simulation-Enhanced Pharmacology Education in Prelicensure Nursing Education. Nurse Educator, 42(5S Suppl 1), S32S37. https://doi.org/10.1097/ NNE.0000000000000409.

Sarfati L, Ranchon F, Vantard N, Schwiertz V, Larbre V, Parat S, ... Rioufol C (2018). Human-simulation-based learning to prevent medication error: A systematic review. Journal of Evaluation in Clinical Practice. https:// doi.org/10.1111/jep.12883

Sears K, O’Brien-Pallas L, Stevens B \& Murphy GT (2013). The Relationship Between the Nursing Work Environment and the Occurrence of Reported Paediatric Medication Administration Errors: A Pan Canadian Study. Journal of Pediatric Nursing, 28(4), 351-356. https://doi.org/10.1016/j. pedn.2012.12.003.

Simonsen BO, Daehlin GK, Johansson I \& Farup PG (2014). Differences in medication knowledge and risk of errors between graduating nursing students and working registered nurses: comparative study. BMC Health Services Research, 14(1), 580. https://doi.org/10.1186/ s12913-014-0580-7. 
Smeulers M, Verweij L, Maaskant JM, de Boer M, Krediet CTP, Nieveen van Dijkum EJM \& Vermeulen $\mathrm{H}$ (2015). Quality Indicators for Safe Medication Preparation and Administration: A Systematic Review. PLOS ONE, 10(4), e0122695. https://doi.org/10.1371/journal. pone.0122695.

Stewart D, Thomas B, MacLure K, Pallivalapila A, El Kassem W, Awaisu A, ... Al Hail M (2018). Perspectives of healthcare professionals in Qatar on causes of medication errors: A mixed methods study of safety culture. PLOS ONE, 13(9), e0204801. https://doi.org/10.1371/journal. pone.0204801.

Toffoletto MC, Canales MA, Moreira D, Ordenes A \& Vergara CA (2015). Errores en la preparación y administración de medicamentos: una revisión integradora de la literatura Latinoamericana. Enfermería Global (Vol. 14). Servicio de Publicaciones, Universidad de Murcia. Disponible en: http://scielo. isciii.es/scielo.php?script=sci_arttext\&pid=S1695-61412015000100016

Valdez LP, de Guzman A \& Escolar-Chua $R$ (2013). A structural equation modeling of the factors affecting student nurses' medication errors. Nurse education today, 33(3), 222-228. https://doi.org/10.1016/j. nedt.2012.01.001.

Wang H-F, Jin J-F, Feng X-Q, Huang X, Zhu L-L, Zhao X-Y \& Zhou Q (2015). Quality improvements in decreasing medication administration errors made by nursing staff in an academic medical center hospital: a trend analysis during the journey to Joint Commission International accreditation and in the post-accreditation era. Therapeutics and clinical risk management, 11, 393-406. https://doi. org/10.2147/TCRM.S79238.

WHO (2009). Patient Safety Curriculum Guide for Medical Schools. (H. W. Organization, Ed.), WHO. France: World Health Organization. Recuperado de http://www.who.int/patientsafety/education/curriculum_guide_ medical_schools/en/.

Xu C, Li G, Ye N \& Lu Y (2014). An intervention to improve inpatient medication management: a before and after study. Journal of Nursing Management, 22(3), 286-294. https://doi. org/10.1111/jonm.12231. 